\title{
Detecting the invisible
}

\section{Carl D. Murray}

IN 1980 the cameras on board Voyager 1 showed the F ring of Saturn to be narrow and dust-rich, and to contain several peculiar features - for example, radial discontinuities (kinks), clumps of ring material and an apparent twisted structure (braids). Two small satellites, Pandora and Prometheus, were discovered, one on each side of the ring. The torques from these moons are generally believed to be responsible for 'shepherding' the ring material, preventing ring spreading. A new analysis of Voyager images by Kolvoord et al., reported on page 695 of this issue ${ }^{1}$, now reveals the tell-tale gravitational signature of Prometheus in the structure of the ring, which may be the cause of its braiding. The analysis also produces evidence for a third satellite at a distance of $1,180 \mathrm{~km}$ from the ring, thereby providing an example of our ability to detect such objects even if they cannot yet be imaged directly.

Dermott ${ }^{2}$ showed that, as well as shepherding ring material, satellites should have the more obvious effect of inducing waves as they pass by. On encountering a satellite, ring particles initially on circular orbits would move to eccentric orbits, all with the same eccentricity but with slightly different phases, giving the appearance of a wave on the ring. The wave would eventually be damped through inter-particle collisions, which make the orbits more circular again. The wavelength of such an induced wave is a function of the distance between the ring and the satellite, and the amplitude is a function both of this distance and of the mass of the satellite. This mechanism was thought to be at least partly responsible for the braided appearance of the ring, through the interactions of the waves produced by both of the shepherding satellites. Prometheus' crossing of the $\mathrm{F}$ ring every 18 years $^{3}$ should also have dramatic consequences for the ring's structure.

Kolvoord et al. report direct evidence of the wave mechanism at work. Although they find no signature from the smaller and more distant satellite Pandora, one set of periodicities in their F-ring images does correspond to that which would be expected from Prometheus. They also claim to have detected the signature of at least one other satellite, although this evidence is less conclusive. A full explanation of all the structure seen in the highest-resolution images (not included in the data set of Kolvoord et al.) will probably require an understanding of the effect of small (radius less than $10 \mathrm{~km}$ ) satellites orbiting within the ring itself. Although the detailed structure of the F ring is complicated, it may prove possible to explain it in terms of the simple gravitational effect of a few nearby satellites.

As further sets of high-resolution images of orbiting material will not be obtained for several years, the detection of satellites and rings by indirect methods has become a necessity. This approach has already met with some success for Saturn. The ring occultation observations made by Voyager revealed very few real gaps, as opposed to density fluctuations, in Saturn's rings. One of the gaps, the 325$\mathrm{km}$-wide Encke division in the broad A ring, has an unusual ringlet, and Cuzzi and Scargle ${ }^{+}$have shown that the edges of the gap have a wave-like appearance at some longitudes. These waves have a much more regular appearance than the braiding seen in the $F$ ring, and Cuzzi and Scargle concluded that they are strongly suggestive of a satellite of $10-\mathrm{km}$ radius orbiting near the centre of the gap. Such a satellite would have been at the threshold of detection by the Voyager cameras. Preliminary studies ${ }^{5}$ of the Keeler gap (width $\sim 38 \mathrm{~km}$ ) near the outer edge of the A ring suggest that the width in fact varies by about $6 \mathrm{~km}$, and that the wavelength of these fluctuations varies with longitude. Again, one or more small satellites orbiting nearby have been invoked to account for the observations.

The rings of Uranus were discovered serendipitously by ground-based occultation in 1977. Even today, most of our knowledge of the uranian ring system has been derived from occultation studies rather than from direct imaging. The first such study of material in orbit around Neptune took place in $1981^{\circ}$. Although occultation events in subsequent years were attributed to the presence of incomplete rings or 'arcs' around the planet, the opaque nature of the 1981 event prompted its observers to maintain that they had detected a satellite. The Voyager flyby of Neptune in August 1989 led to the discovery of six new satellites, one of which (temporarily named $1989 \mathrm{~N} 2$ ) was of the right size (radius $\approx 100 \mathrm{~km}$ ) and in the right orbit to explain the 1981 event $^{7}$, confirming that the satellite had indeed been first discovered by occultation.

Perhaps the most unexpected source of information on the locations of rings and satellites consists of charged-particle experiments. Rings and satellites create 'shadows' or 'wakes' in the plasma environment around planets. Observations by Pioneer 11 at Saturn showed reductions in the particle flux at various locations; some could be identified with the orbits of known satellites and rings, but others were attributed to material not previously known. This led to the discovery of Saturn's G ring and confirmed the existence of the satellite Janus, both of which were subsequently imaged by the Voyager spacecraft.

Carl D. Murray is in the Astronomy Unit, Queen Mary and Westfield College, Mile End Road. London E1 $4 N S$, UK.

1. Kolvoord, R.A., Burns, J.A. \& Showalter, M.R. Nature 345 695-697 (1990)

2. Dermott, S.F. Nature 290, 454-457 (1981)

3. Borderies, N., Goldreich, P. \& Tremaine, S. Icarus $\mathbf{5 3}$ 84-89 (1983)

4. Cuzzi, J. \& Scargle, J.D. Astrophys, J. 292, 276-290 (1985).

5. Cooke, M.L., Nicholson, P.D. \& Showalter, M.R. Bull. Am astr. Soc. 21, 928 (1989).

6. Reitsema, H.J. et al. Science 215, 289-291 (1982) 7. Smith, B.A. et al. Science 246, 1422-1449 (1989).

\section{Fruits for the taking}

\section{Peter D. Moore}

GETTING from one place to another can be a serious problem for rooted plants. But being the first to arrive at a suitable location gives undoubted competitive advantages, hence the wide range of fruitdispersal mechanisms found in the plant world. The structural mechanics of plumed fruits provides a seemingly unlimited source of data for aerodynamic analysis and the assessment of transport efficiencies ${ }^{1}$, but the evolutionary relationship between fruits and their vertebrate dispersal vectors has stimulated even more ecological research projects. The search continues for a general relationship between fruit size, morphology and chemistry, and the bills, gapes, jaws, maws and guts of their bird and mammal consumers. Some fairly broad conclusions are beginning to emerge.
Generally, fruit-eating birds are large and have a relatively wide gape, as has been demonstrated in temperate areas ${ }^{2}$, the Mediterranean region ${ }^{3}$ and tropical forests ${ }^{4}$. For their area of study (near Montpellier in France), Debussche and Isenmann $^{3}$ report the results of an eightyear analysis of vertebrate involvement in fruit dispersal and show that there is a strong positive correlation between the gape size of birds and the volume of the fruits they feed upon. There is a similarly positive relationship between the disperser weight (both birds and mammals) and fruit volume.

The second finding is reminiscent of the relationship between predators and their prey, but there are marked differences in the case of fruit-eating, as Debussche and Isenmann point out. In the case of fruit, it 